\title{
The distribution of triage categories and the impact of emergency symptoms and signs on the triage level
}

\author{
Charlotte Barfod ${ }^{1 *}$, Jakob Danker ${ }^{1}$, Jakob Forberg ${ }^{2}$, Marlene MP Lauritzen ${ }^{2}$ \\ From Danish Society for Emergency Medicine: Research Symposium 2010 \\ Roskilde, Denmark. 20-21 May 2010
}

\section{Background}

The Emergency Department (ED) at Hillerød Hospital uses a five-level triage system inspired by the Swedish ADAptiv Process Triage (ADAPT). The triage categories are red, orange, yellow, green and blue. Patients classified as red need immediate treatment whereas blue patients are non-urgent and not admitted to hospital. The patient is assigned a triage category based on both vital signs and on "emergency symptoms and signs" (ESS).The highest level of the two determines the overall triage category.

\section{Methods}

During a 6 months period, all patients admitted to the ED were registered consecutively and triaged according to the triage model. The patients were triaged at three different time points: 1 ) at the time of admission to the ED; 2) during reassessment; 3) before transfer to another department or discharge. The following data were registered at each time point: triage time, vital signs (respiratory rate, peripheral saturation by pulse oxymetry, blood pressure, pulse, Glasgow Coma Score and temperature), chief complaint and triage category. All data were entered into a data base specifically designed for the study.

\section{Results}

In the period 1st of September 2009 to 1st of March 201020.409 patients attended the ED of which 6.911 patients were admitted and registered in the database. At the time of ED admission patients were triaged in red, orange, yellow and green in $3.1 \%, 22.7 \%, 42.7 \%$ and
$31.5 \%$ of the cases, respectively. The most common ESS algorithm was the gastrointestinal complaint algorithm (20.3\%) followed by chest pain (8.3\%), dyspnoea (8.2\%) and neurological complaint (5.9\%). In 37\% the ESS algorithm resulted in an upgrade of the triage category.

\section{Conclusion}

Triage level yellow (needing urgent treatment) was the most common triage category in patients admitted to the ED at a larger Danish hospital. A minority of the patients needed immediate treatment (triage level red). In more than one third of the patients the chief complaint resulted in an upgrade of the triage category. The distribution of triage categories are important in order to plan for the future organisation of the ED.

\section{Author details}

'Department of Anaesthesiology, Hillerød Hospital, Denmark. ${ }^{2}$ Department of Emergency Medicine, Hillerød Hospital, Denmark.

Published: 17 September 2010

doi:10.1186/1757-7241-18-S1-P34

Cite this article as: Barfod et al: The distribution of triage categories and the impact of emergency symptoms and signs on the triage level. Scandinavian Journal of Trauma, Resuscitation and Emergency Medicine 2010 18(Suppl 1):P34.

* Correspondence: cbar@hih.regionh.dk

'Department of Anaesthesiology, Hillerød Hospital, Denmark

Full list of author information is available at the end of the article 